\title{
OTIMIZAÇÃO DO DESCASCAMENTO QUÍMICO DE RAÍZES DO YACON (Polymnia sonchifolia Poepp.)
}

\author{
Optimization of chemical peeling of yacon roots (Polymnia sonchifolia Poepp.)
}

\author{
Elaine Berges da Silva ${ }^{1}$, Rui Sérgio dos Santos Ferreira da Silva ${ }^{3}$, \\ Laura Beatriz Karam², Lys Mary Bileski Cândido ${ }^{4}$
}

\begin{abstract}
RESUMO
Foi utilizado o delineamento fatorial completo $2^{3}$ para estudar o efeito das variáveis de processo no descascamento químico de raízes do yacon, cultivadas na região metropolitana de Curitiba, estado do Paraná. Foram realizados 11 experimentos, com três repetições no ponto central, nos quais foram avaliados em três níveis a concentração da solução de hidróxido de sódio, \% (g/100 mL) $[6,10,14]$, temperatura da solução de hidróxido de sódio, ${ }^{\circ} \mathrm{C}[70,80,90]$ e tempo de permanência na solução de hidróxido de sódio, minutos $[2,4,6]$. Todas as variáveis estudadas afetaram de forma significativa $(\mathrm{p}<0,05)$ o rendimento das raízes submetidas ao descascamento químico. A variável mais importante para o rendimento foi o tempo de permanência na solução de hidróxido de sódio. O modelo obtido para a resposta percentual de rendimento foi adequado, $\operatorname{com} \mathrm{R}^{2} \mathrm{aj}=0,8497$ e com falta de ajuste não significativa $(\mathrm{p}=$ 0,9312), podendo ser utilizado para fins preditivos. No ponto central, foi obtido um rendimento satisfatório $(84 \%$ a $87 \%)$ e elevado percentual de casca removida ( $96 \%$ a 98\%), indicando que o tratamento com $10 \%$ de solução de hidróxido de sódio a temperatura de $80^{\circ} \mathrm{C}$ por 4 minutos, pode ser empregado no descascamento químico das raízes do yacon.
\end{abstract}

Termos para indexação: Descascamento químico, delineamento fatorial, lixívia, Smallanthus sonchifolia.

\section{ABSTRACT}

A full factorial design $2^{3}$ was used to evaluate the effect of process variables in chemical peeling of yacon roots, cultivated in Curitiba, State of Paraná. Eleven treatments, with three central points, were done in which they had been evaluated at three different levels of sodium hydroxide solution, $\%(\mathrm{~g} / 100 \mathrm{~mL})[6,10,14]$, temperature of the same solution, ${ }^{\circ} \mathrm{C}[70,80,90]$, and residence time in the sodium hydroxide solution, minutes $[2,4,6]$. All the studied variables had affected significantly $(\mathrm{p}<0.05)$ the yield of yacon roots subjected to chemical peeling. The variable that most affected the yield was the time of permanence in the sodium hydroxide solution. The mathematical model obtained for the yield $(\%)$ was good with $\mathrm{R}^{2}$ aj $=0.8497$, and non significant lack of fit $(\mathrm{p}=0.9312)$.Therefore, the model can be used for predictive purposes. In the central point a satisfactory yield $(84 \%$ to $87 \%)$ with a high percentage of removed peel was obtained (96\% to $98 \%$ ) indicating that the treatment with $10 \%$ of sodium hydroxide solution, temperature of $80^{\circ} \mathrm{C}$ per 4 minutes can be used in the chemical peeling of yacon roots.

Index terms: Chemical peeling, factorial design, lixyvium, Smallanthus sonchifolia.

(Recebido em 25 de julho de 2007 e aprovado em 19 de março de 2009)

\section{INTRODUÇÃO}

O yacon é uma planta de origem andina, do gênero Polymnia L., pertencente à família Asteraceae Bercht. \& J. Presl ou Compositae Giseke (Zardini, 1991). Introduzido no Brasil na década de 90 , o yacon vem despertando o interesse do mundo científico, em decorrência de seu potencial como alimento funcional. Diferente da maioria das raízes que armazenam carboidratos na forma de amido, o yacon e várias plantas da família Compositae armazenam os carboidratos na forma de frutanos. Os órgãos subterrâneos do yacon contêm de 60 a $70 \%$ de frutanos do tipo inulina com grau de polimerização (GP) máximo de 12 (Vilhena et al., 2000).

$\mathrm{Na}$ medicina popular, as folhas e raízes do yacon são consumidas in natura ou desidratadas, na forma de chá, contra diabetes e altas taxas de colesterol (Vilhena et al., 2000). Entre as numerosas opções que oferece o yacon, a produção de bebidas aparece como uma das mais interessantes. Na Europa o mercado de bebidas funcionais foi estimado em, $7 \%$ do mercado de refrigerantes no ano de 2004, com aumento de $8 \%$ em 2005. De acordo com as previsões o consumo deve atingir 5,1 bilhões de litros até o ano de 2009, o que corresponde a um aumento de $23 \%$ comparado com 2005 (Siró et al., 2008).

O yacon, na forma de bebida, suco ou néctar, é de fácil comercialização e consumo podendo ser incorporado à dieta dos diabéticos e da população em geral. A importância de frutas processadas vem crescendo, em virtude das facilidades proporcionadas pelos produtos já

\footnotetext{
1Universidade Federal do Paraná/UFPR - Curitiba, PR

${ }^{2}$ Universidade Estadual de Londrina/UEL - Curitiba, PR

${ }^{3}$ Universidade Estadual de Campinas/UNICAMP - Londrina, PR

${ }^{4}$ Universidade Estadual de Campinas/UNICAMP - Curitiba, PR - Ivsmarv@ufpr.br
} 
prontos para o consumo, do maior número de pessoas morando sozinhas e da melhoria da qualidade dos produtos ofertados (Damiani et al., 2009). Para a obtenção de bebidas à base do yacon, as raízes in natura, previamente branqueadas são prensadas para a extração do suco, o qual pode ser misturado ao suco de outras frutas ou vegetais e adoçados com açúcar ou com edulcorantes quando destinados ao consumo por diabéticos.

Além das propriedades promotoras de saúde, o yacon também pode ser usado para melhorar aspectos sensoriais em produtos de panificação de baixo valor calórico (Moscatto et al., 2004).

De aparência similar à batata doce, as raízes tuberosas do yacon são fusiformes, apresentando grande variação no tamanho, formato e peso (Butler \& Rivera, 2004). A falta de uniformidade das raízes inviabiliza o uso de equipamentos industriais normalmente utilizados no processamento de vegetais, como os processos por abrasão. Dessa forma, o descascamento das raízes do yacon pode ser realizado manualmente ou quimicamente, empregando-se solução de hidróxido de sódio (lixívia) à quente. No descascamento químico o rendimento varia conforme o tratamento empregado, sendo inversamente proporcional ao aumento na concentração, temperatura e tempo de permanência na lixívia, indicando que percentuais mais elevados de rendimento podem ser obtidos em condições menos drásticas de tratamento. O inverso ocorre com o percentual de casca removida, gerando a necessidade de retrabalho para a remoção manual da casca remanescente (Garrote et al., 1993).

O antagonismo observado para as respostas rendimento e percentual de casca removida aponta para a necessidade de metodologias que otimizem simultaneamente as duas variáveis. Com base nas considerações apresentadas, neste trabalho, objetivou-se otimizar o processo de descascamento químico de raízes do yacon, por meio de estudo do efeito dos fatores concentração e temperatura da solução de hidróxido de sódio e tempo de permanência das raízes do yacon nestas soluções.

\section{MATERIAL E MÉTODOS}

\section{Material}

Foram avaliadas 110 raízes do yacon in natura (Polymnia sonchifolia Poepp.), cultivadas na região metropolitana de Curitiba. As raízes recém-colhidas foram lavadas em água corrente, imersas em água clorada 10 ppm por 10 minutos e o excesso de água foi escorrido. Para se realizar uma distribuição homogênea das raízes em cada tratamento, as raízes foram divididas em 4 classes, de acordo com a área superficial, determinada conforme descrito por
Garrote et al. (1993). A partir dessa classificação, as raízes foram subdivididas em 11 lotes contendo 8 raízes cada, de forma que cada lote contivesse a mesma distribuição de frequências observada para cada classe.

Para o descascamento químico foi utilizada soda cáustica comercial ( $98 \%$ de pureza) e ácido cítrico de grau analítico.

\section{Descascamento Químico}

Os experimentos foram realizados na planta de Processamento de Vegetais na Usina Piloto do curso de Engenharia de Alimentos da Pontifícia Universidade Católica do Paraná (PUC-PR). As amostras foram tratadas pela submersão em solução de hidróxido de sódio (lixívia) pelo tempo determinado para cada tratamento, lavadas em água corrente e mergulhadas em solução de ácido cítrico 3\% por 30 segundos para neutralização do $\mathrm{pH}$. Os tratamentos foram realizados de forma aleatorizada, utilizando delineamento fatorial completo $2^{3}$ adotando as seguintes variáveis independentes (fatores) em 3 níveis de variação: concentração da lixívia, \% (g/100 mL) $[6,10,14]$, temperatura da lixívia, ${ }^{\circ} \mathrm{C}[70,80,90]$, e tempo de permanência na lixívia, minutos $[2,4,6]$. O experimento foi composto por 11 tratamentos, sendo 3 foram repetições do ponto central. As variáveis dependentes (respostas) estudadas foram os percentuais de rendimento e de casca removida.

Para a determinação da porcentagem de casca removida foi realizada a avaliação da área superficial da raiz antes do descascamento químico e da área remanescente após o descascamento, conforme metodologia descrita por Garrote et al. (1993). A determinação da porcentagem de rendimento foi realizada com base no peso da raiz antes e após o descascamento químico, realizando-se a raspagem da casca remanescente com uma faca. Os resultados obtidos foram analisados estatisticamente.

\section{Análise Estatística}

Foi utilizado o delineamento fatorial completo $2^{3}$, no qual a função que vincula as variáveis dependentes com as variáveis independentes, de acordo com Barros Neto et al. (2002) é um modelo multilinear, que tem a seguinte forma:

$\mathrm{Y}\left(\mathrm{x}_{1}, \mathrm{x}_{2}, \mathrm{x}_{3}\right)=\beta_{0}+\beta_{1} \mathrm{x}_{1}+\beta_{2} \mathrm{x}_{2}+\beta_{3} \mathrm{x}_{3}$

Onde:

$\mathrm{x}_{1}, \mathrm{x}_{2}$ e $\mathrm{x}_{3}$ são as variáveis independentes que, neste estudo, são respectivamente a concentração da lixívia, temperatura da lixívia e tempo de permanência da lixívia e;

$\beta_{0}, \beta_{1}, \beta_{2} \beta_{3}$ são os coeficientes de regressão obtidos com o uso do modelo. 
Os resultados obtidos foram analisados estatisticamente, utilizando o programa Statistica 6.0 da Statsoft, em sistema Windows, para a determinação dos coeficientes dos polinômios e a análise de variância (Statistica..., 2002).

\section{RESULTADOS E DISCUSSÃO}

O percentual de rendimento variou de $73,84 \%$ a $92,50 \%$, respectivamente, para as condições mais drásticas de tratamento (maior concentração da lixívia, maior temperatura e maior tempo de permanência) e menos drásticas (menor concentração da lixívia, menor temperatura e menor tempo de permanência na lixívia), conforme apresentado na Tabela 1 .

Os resultados da análise de variância para o percentual de rendimento mostram que as três variáveis de processo estudadas foram significativas $(p<0,05)$, conforme apresentado na Tabela 2. O modelo obtido é multilinear, com boa qualidade de ajustamento, isto é, a falta de ajuste não foi significativa $(\mathrm{p}=0,93)$.

A equação que foi ajustada aos dados experimentais está apresentada abaixo, demonstrando que a variável que mais afetou a resposta foi o tempo de permanência na lixívia, apresentando o coeficiente com maior valor absoluto. Os coeficientes negativos obtidos para todas as variáveis explicam a redução do percentual de rendimento nas condições mais drásticas de tratamento, conforme discutido anteriormente. Resultados semelhantes foram obtidos por Garrote et al. $(1993,1998)$ para o descascamento químico de batatas e aspargos.

A modelagem mostrou-se adequada com $R^{2}$ aj $=$ 0,8497 (coeficiente de determinação ajustado) e baixo coeficiente de variação $\mathrm{CV}=2,51 \%$, podendo ser utilizada para fins preditivos.

Na Tabela 3, apontam-se os resultados da análise de variância para o percentual de casca removida, demonstrando que o modelo obtido não pode ser utilizado para fins preditivos, apresentando falta de ajuste significativa $(p=0,0010)$, baixo $R^{2} a j=0,5727$ e alto $C V=$ $28,88 \%$. Modelos mais complexos, que consideram as interações entre os fatores, também não apresentaram boa capacidade preditiva.

$$
\% \text { Rendimento }\left(\mathrm{X}_{1}, \mathrm{X}_{2}, \mathrm{X}_{3}\right)=84,31364-2,24125 \mathrm{X}_{1}-2,25625 \mathrm{X}_{2}-4,84125 \mathrm{X}_{3}
$$

Onde:

$\mathrm{X}_{1}=$ concentração da lixívia, $\%$ ou g/100 mL

$\mathrm{X}_{2}=$ temperatura da lixívia, ${ }^{\circ} \mathrm{C}$

$\mathrm{X}_{3}=$ tempo de permanência na lixívia, min

Tabela 1 - Percentual de rendimento e de remoção de casca no descascamento químico de raízes do yacon.

\begin{tabular}{ccccccccc}
\hline $\begin{array}{c}\text { Experimento } \\
\text { Ordem Padrão }\end{array}$ & \multicolumn{2}{c}{$\begin{array}{c}\text { Delineamento } \\
\text { Estatístico }\end{array}$} & $\begin{array}{c}\text { Concentração } \\
\text { Lixívia }(\%)\end{array}$ & $\begin{array}{c}\text { Temperatura } \\
\text { Lixívia }\left({ }^{\circ} \mathrm{C}\right)\end{array}$ & $\begin{array}{c}\text { Tempo } \\
\text { Lixívia (min) }\end{array}$ & $\begin{array}{c}\text { Rendimento } \\
(\%)\end{array}$ & $\begin{array}{c}\text { Casca } \\
\text { Removida (\%) }\end{array}$ \\
\hline 1 & $\mathrm{X} 1$ & $\mathrm{X} 2$ & $\mathrm{X} 3$ & $\mathrm{X} 1$ & $\mathrm{X} 2$ & $\mathrm{X3}$ & & \\
\hline 2 & -1 & -1 & -1 & 6 & 70 & 2 & 92,50 & 15,27 \\
3 & +1 & -1 & -1 & 14 & 70 & 2 & 88,65 & 30,73 \\
4 & -1 & +1 & -1 & 6 & 90 & 2 & 90,38 & 35,52 \\
5 & +1 & +1 & -1 & 14 & 90 & 2 & 85,98 & 94,99 \\
6 & -1 & -1 & +1 & 6 & 70 & 6 & 85,31 & 58,47 \\
7 & +1 & -1 & +1 & 14 & 70 & 6 & 80,71 & 98,78 \\
8 & -1 & +1 & +1 & 6 & 90 & 6 & 78,92 & 98,28 \\
9 & +1 & +1 & +1 & 14 & 90 & 6 & 73,84 & 99,52 \\
10 & 0 & 0 & 0 & 10 & 80 & 4 & 84,02 & 96,51 \\
11 & 0 & 0 & 0 & 10 & 80 & 4 & 80,36 & 96,16 \\
\hline
\end{tabular}


Tabela 2 - Análise de variância para a variável resposta percentual de rendimento do descascamento.

\begin{tabular}{lccccc}
\hline \multicolumn{1}{c}{ EFEITOS } & $\begin{array}{c}\text { SOMA DOS } \\
\text { QUADRADOS }\end{array}$ & $\begin{array}{c}\text { GRAUS DE } \\
\text { LIBERDADE }\end{array}$ & $\begin{array}{c}\text { QUADRADO } \\
\text { MÉDIO }\end{array}$ & VALOR F & VALOR P \\
\hline Concentração lixívia (\%) & 40,18 & 1 & 40,18 & 8,9150 & $0,0203^{*}$ \\
Temperatura lixívia $\left({ }^{\circ} \mathrm{C}\right)$ & 40,72 & 1 & 40,72 & 9,0347 & $0,0197^{*}$ \\
Tempo lixívia (min) & 187,50 & 1 & 187,50 & 41,5966 & $0,0003^{*}$ \\
Resíduo & 31,55 & 7 & 4,50 & & \\
Falta de ajuste & 10,81 & 5 & 2,16 & 0,2084 & 0,9312 \\
Erro experimental & 20,74 & 2 & 10,37 & & \\
Total & 299,96 & 10 & & & \\
$\mathrm{R}^{2}$ & 0,8948 & & & & \\
$\mathrm{R}^{2}$ ajustado & 0,8497 & & & & \\
\hline
\end{tabular}

NOTA: $(*)$ estatisticamente significativa em nível de $5 \%$ de probabilidade.

Tabela 3 - Análise de variância para a variável resposta percentual de casca removida.

\begin{tabular}{lccccc}
\hline \multicolumn{1}{c}{ EFEITOS } & $\begin{array}{c}\text { SOMA DOS } \\
\text { QUADRADOS }\end{array}$ & $\begin{array}{c}\text { GRAUS DE } \\
\text { LIBERDADE }\end{array}$ & $\begin{array}{c}\text { QUADRADO } \\
\text { MÉDIO }\end{array}$ & VALOR F & VALOR P \\
\hline Concentração lixívia (\%) & 1695,95 & 1 & 1695,95 & 3,6429 & $0,0979^{*}$ \\
Temperatura lixívia $\left({ }^{\circ} \mathrm{C}\right)$ & 1955,00 & 1 & 1955,00 & 4,1993 & $0,0796^{*}$ \\
Tempo lixívia (min) & 3984,57 & 1 & 3984,57 & 8,5587 & $0,0221^{*}$ \\
Resíduo & 3258,88 & 7 & 465,55 & & \\
Falta de ajuste & 3257,50 & 5 & 651,50 & 946,0770 & $0,0010^{*}$ \\
Erro experimental & 1,38 & 2 & 0,689 & & \\
Total & 10894,39 & 10 & & & \\
$\mathrm{R}^{2}$ & 0,7009 & & & & \\
$\mathrm{R}^{2}$ ajustado & 0,5727 & & & & \\
\hline
\end{tabular}

NOTA: $(*)$ estatisticamente significativa em nível de 5\% de probabilidade.

Nas condições mais drásticas de tratamento, além da casca, parte da polpa também foi removida justificando o menor percentual de rendimento obtido nessas condições. Isso pode ser confirmado pelos resultados obtidos para o percentual de casca removida que apresentou resultados inversamente proporcionais aos obtidos para o percentual de rendimento, demonstrando que essas respostas devem ser analisadas conjuntamente. Deve-se buscar, portanto, uma condição de tratamento que otimize as duas variáveis dependentes, resultando em um elevado percentual de rendimento e, ao mesmo tempo, um elevado percentual de casca removida, evitando, dessa forma, a necessidade de remoção manual da casca remanescente após o tratamento.

Resultados satisfatórios foram obtidos no ponto central (Tabela 1) que apresentou bom rendimento (cerca de $84 \%$ ) e elevado percentual de casca removida (cerca de $97 \%$ ), indicando que o tratamento com solução de hidróxido de sódio à $10 \%$, à temperatura de $80^{\circ} \mathrm{C}$ por 4 minutos, pode ser empregado para o descascamento químico das raízes do yacon. Garrote et al. (1993) obtiveram resultados semelhantes para o descascamento de batatas, no qual o tratamento em solução de hidróxido de sódio $12,72 \%$, temperatura de $95^{\circ} \mathrm{C}$ e tempo de 5,67 minutos resultaram em $80 \%$ de rendimento.

\section{CONCLUSÕES}

As variáveis concentração da solução de hidróxido de sódio, temperatura da solução de hidróxido de sódio e tempo de permanência na solução de hidróxido de sódio, afetaram de forma significativa $(\mathrm{p}<0,05)$ o rendimento das raízes submetidas ao descascamento químico. A variável 
que mais afetou o rendimento foi o tempo de permanência na solução de hidróxido de sódio.

O modelo obtido para a variável percentual de rendimento foi adequado, com $R^{2}$ aj $=0,8497$ e falta de ajuste não significativa $(\mathrm{p}=0,9312)$, podendo ser utilizado para fins preditivos.

No ponto central, foi obtido um rendimento satisfatório ( $84 \%$ a $87 \%$ ) e elevado percentual de casca removida (96\% a $98 \%$ ), indicando que o tratamento com $10 \%$ de solução de hidróxido de sódio à temperatura de $80^{\circ} \mathrm{C}$ por 4 minutos, pode ser empregado no descascamento químico das raízes do yacon.

\section{REFERÊNCIAS BIBLIOGRÁFICAS}

BARROS NETO, B.; SCARMINIO, I.S.; BRUNS, R.E. Como fazer experimentos: pesquisa e desenvolvimento na ciência e na indústria. 2.ed. Campinas: Unicamp, 2002. 401p.

BUTLER, G.; RIVERA, D. Innovations in peeling technology for yacon. Lima: International Potato Center, 2004. 15p. Disponível em: <http://www.cipotato.org/artc ccip_crops/2004-1127.pdfì. Acesso em: 1 set. 2010.

DAMIANI, C.; VILAS-BOAS, E.V.B.; SOARES JUNIOR, M.; CALIARI, M.; PAULA, M.L. de; ASQUIERI, E.R. Avaliação química de geléias de manga formuladas com diferentes níveis de casca em substituição à polpa. Ciência e Agrotecnologia, Lavras, v.33, n.1, p.177-184, jan./fev. 2009.
GARROTE, R.L.; COUTAZ, V.R.; LUNA, J.A.; SILVA, E.R.; BERTONE, R.A. Optmizing processing conditions for chemical peeling of potatoes using response surface methodology. Journal of Food Science, Champaign, v.58, n.4, p.821-926, 1993.

GARROTE, R.L.; SILVA, E.R.; BERTONE, R.A.; AVALLE, A. Pelado quimico y termofisico de esparragos. Ciência de Tecnologia de Alimentos, Campinas, v.18, n.3, p.319324, 1998 .

MOSCATTO, J.A.; PRUDÊNCIO-FERREIRA, S.H.; HAULY, M.C. Farinha de yacon e inulina como ingredientes na formulação de bolo de chocolate.

Ciência de Tecnologia de Alimentos, Campinas, v.24, n.4, p.634-640, 2004.

SIRÓ, I.; KÁPOLNA, E.; KÁPOLNA, B.; LUGASI, A. Functional food: product development, marketing and consumer acceptance: a review. Appetite, v.51, p.456-467, 2008.

STATISTICA for Windows. Versão 6.0. Tulsa: Statsoft, 2002. v.4. Software.

VILHENA, S.M.C.; CÂMARA, F.L.; KADIHARA, S.T. O cultivo do yacon no Brasil. Horticultura Brasileira, Brasília, v.18, n.1, p.5-8, 2000.

ZARDINI, E. Ethnobotanical notes of yacon, Polymnia sonchifolia (Asteraceae). Economic Botany, v.45, n.1, p.72-85, 1991. 